\title{
Periodic neural activity induced by network complexity
}

\author{
D. R. Paula, ${ }^{1}$ A. D. Araújo, ${ }^{1}$ J. S. Andrade, Jr., ${ }^{1}$ H. J. Herrmann,,${ }^{1,2}$ and J. A. C. Gallas ${ }^{3}$ \\ ${ }^{1}$ Departamento de Física, Universidade Federal do Ceará, 60451-970 Fortaleza, Brazil \\ ${ }^{2}$ Institute for Computational Physics, Universität Stuttgart, D-70569 Stuttgart, Germany \\ ${ }^{3}$ Instituto de Física, Universidade Federal do Rio Grande do Sul, 91501-970 Porto Alegre, Brazil
}

(Received 14 March 2006; published 20 July 2006)

\begin{abstract}
We study a model for neural activity on the small-world topology of Watts and Strogatz and on the scale-free topology of Barabási and Albert. We find that the topology of the network connections may spontaneously induce periodic neural activity, contrasting with nonperiodic neural activities exhibited by regular topologies. Periodic activity exists only for relatively small networks and occurs with higher probability when the rewiring probability is larger. The average length of the periods increases with the square root of the network size.

DOI: 10.1103/PhysRevE.74.017102

PACS number(s): 05.45.Xt, 05.50.+q, 89.75.Da, 89.75.Fb
\end{abstract}

The human brain is the most fascinating processor, consisting of about $10^{10}$ neurons. These neurons are connected to each other by synapses, forming together the neural network. The synapses transmit stimuli through different concentrations of $\mathrm{Na}^{+}$and $\mathrm{K}^{+}$ions. The neurons communicate with each other through electrical impulses. Each time a neuron is charged beyond a certain threshold by the connected neurons, it "fires" an electrical discharge through its axon which through synapses transmits charges to the dendrites of other neurons. While most synapses just connect nearby neurons, a few of them also can be long range and connect to a neuron in a distant area of the brain. These few far-reaching connections seem to be crucial for the coherent functioning of the brain. Such a mixed network structure of many shortand a few long-range connections is the trademark of smallworld networks as introduced in a seminal work by Watts and Strogatz [1-3]. In vitro studies of neuronal networks have in fact been grown and analyzed and found to have small-world properties [4]. Another direction of research has been analyzing avalanches of neurons firing, and it has been reported that there exists some criticality or scale-free behavior. This has been observed experimentally in organotypic cultures from coronal slices of rat cortex [5] and been modeled as a self-organized critical process [6].

In brain research, the appearance of periodic cycles of firing sequences is commonly observed, and is considered as responsible for the origin of various body clocks; it has even been interpreted as the realization of some simple thoughts. One of the fundamental questions is how does such a seemingly disordered system as the brain synchronize the activity so as to produce these periodic signals. It is the aim of the present work to present a simple neural model on WattsStrogatz and Barabási-Albert networks and show that it can, under suitable circumstances, spontaneously generate periodic time series in its activity. Similar studies have already been performed using the Hodgkin-Huxley model [7-9] where it was found that the Watts-Strogatz network has fast coherent oscillations as opposed to other types of graphs. Also integrate-and-fire neurons have been studied on smallworld networks and a transition between self-sustained persistent activity and failure has been reported [10]. Other properties like, for instance, the background of the neural activity, seem rather unaffected by the small-world properties as observed recently [11]. We also want to take into account scale-free properties by investigating our model on the network of Barabási and Albert [12,13]. Among others, the Hopfield model $[14,15]$ and the Hindmarsh-Rose neural model [16] have already been studied before on such networks.

In order to describe the neural activity we use a variant of the original model of McCulloch and Pitts [17]. A neuron $i$ can be in one of two states, firing or nonfiring, described by binary variables $x_{i}=1$ (active) or 0 (inactive). They are initialized randomly. The state of a neuron is updated in time $t$ through the following equation [18]:

$$
x_{i}(t)=\Theta\left(\sum_{j \in(i)}^{n} S_{i j} x_{j}(t-1)+T\right),
$$

where $n$ is the number of connections of each neuron and $S_{i j}$ represents the strength of the synapse between neurons $i$ and $j$. The strength factors $S_{i j}$ are set randomly to be either +1 or -1 with equal probability, representing either an excitatory or an inhibitory neuron, respectively. In reality, the synaptic strengths are not time independent, as known since the seminal work of Bernard Katz, but we consider that these changes occur on much longer time scales than the oscillations we consider in this study. Finally, the variable $T$ is the firing threshold which in fact throughout this paper is taken to be zero. Here, $\Theta$ denotes the Heaviside function defined as usual: $\Theta(z)=1$ if $z \geqslant 0$, and $\Theta(z)=0$ if $z<0$.

We are interested in analyzing the overall firing activity of the brain which in practice can be monitored, for instance, through electroencephalogram (EEG) measurements. For that we define a macroscopic variable of our model which we call the "neural activity" $A(t)$ as the fraction of neurons firing at time $t$,

$$
A(t)=\frac{1}{N} \sum_{i=1}^{N} x_{i}(t)
$$

Note that it is easy to reduce the overall activity in our model by multiplying the integration at each neuron with a constant less than unity. However this will only change the time scale making the program slower and yielding the same result.

The small-world network of Watts and Strogatz [1-3] allows one to continuously connect two extreme situations, 

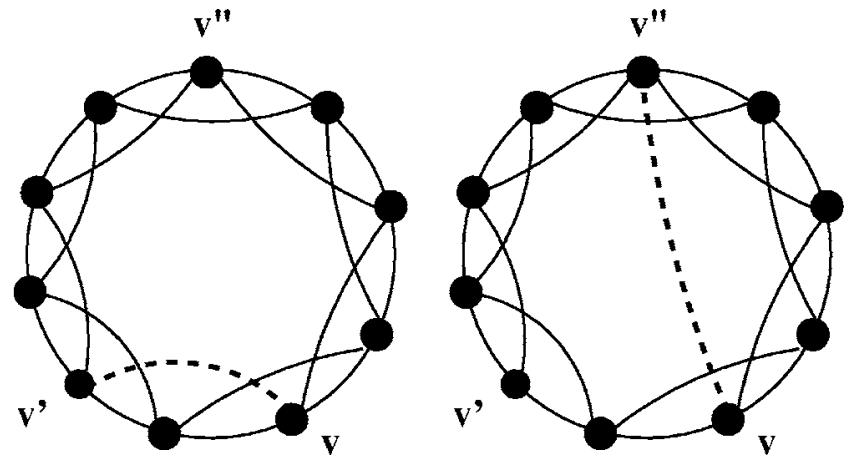

FIG. 1. One starts with a regular chain having nearest- and next-nearest-neighbor connections. With probability $p$ we replace short-range by long-range connections by rewiring. Initially $v$ is connected to $v^{\prime}$. But after rewiring, the connection of $v$ to $v^{\prime}$ is replaced by another connection, say $v$ to $v^{\prime \prime}$.

namely, the regular lattice and random graph of Renyi and Erdös [19]. The construction of a Watts-Strogatz network is performed in two steps.

(1) We start with a regular lattice, in our case a onedimensional chain of $N$ sites with connections between nearest and next-nearest neighbors and periodic boundary conditions so that the total number of connections per site is $k$ $=4$.

(2) With probability $p$ ("rewiring probability") we replace for each site $i$ a connection $L_{i j}$ by another one $L_{i x}$ where $x$ is any randomly chosen site (Fig. 1).

The rewiring probability $p$ varies between zero and one and is the main parameter of our investigation. For $p=0$ we have a regular lattice and for $p=1$ a random graph.

The scale-free network of Barabási and Albert $[12,13]$ is constructed by starting with a small number $m_{0}$ of nodes at time $t=0$. Then, at each time step one adds a new node having $m<m_{0}$ links to the existing nodes. The probability that a new node is connected to node $i$ is $k_{i} / \sum_{j} k_{j}$ where $k_{i}$ is the actual connectivity of node $i$.

This rule assures preferential attachment to sites of higher connectivity. As a result the distribution of connectivities also called the "degree distribution" follows a power law $P(k) \sim k^{-\gamma}$ with an exponent $\gamma=3$. This property characterizes a scale-free network.

We first consider a network with $N=2048$ sites and modify the rewiring probability $p$ between zero and one. For each value of $p$, we generate 1000 different networks and compute at each time step the neural activity $A(t)$, Eq. (2), as generated by the model of Eq. (1). Figure 2(a) shows the nonperiodic temporal evolution of the neural activity as obtained for a Watts-Strogatz network with rewiring probability $p=0$ (i.e., on a regular chain). In order to measure the periodicity in time, we analyze the last 1024 time steps of series with 16382 steps using a shift algorithm In this way, it is possible to detect all periods shorter than 512 time steps.

Figure 2(b) shows the result when the rewiring probability is $p=1$ (i.e., on a random graph): the time series becomes periodic. To quantify the degree of periodicity $\phi$, i.e., the fraction of graphs that exhibit periodic time series, we performed additional simulations on networks with $N=1024$,

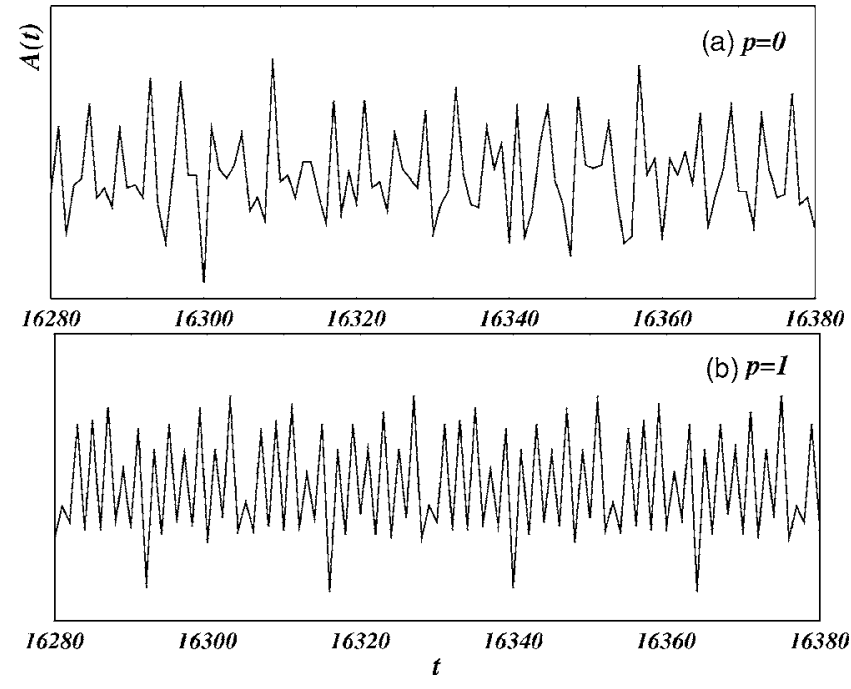

FIG. 2. Temporal evolution of the neural activity of graphs having 2048 sites (a) for a rewiring probability $p=0$, i.e., a regular network; (b) for a rewiring probability $p=1$, i.e., a random graph.

2048, 4096, 8192, and 16384 sites. For each size we generated 1000 different networks and measured the degree of periodicity for various values of $p$.

The inset of Fig. 3 shows the fraction $\phi$ of networks reaching a periodic regime as a function of the rewiring probability $p$. For $p=0$ we find consistently $\phi=0$, i.e., all networks behave non-periodically regardless of their size. Furthermore, the solid lines clearly indicate that the increase of the fraction $\phi$ with $p$ can be closely described by the expression,

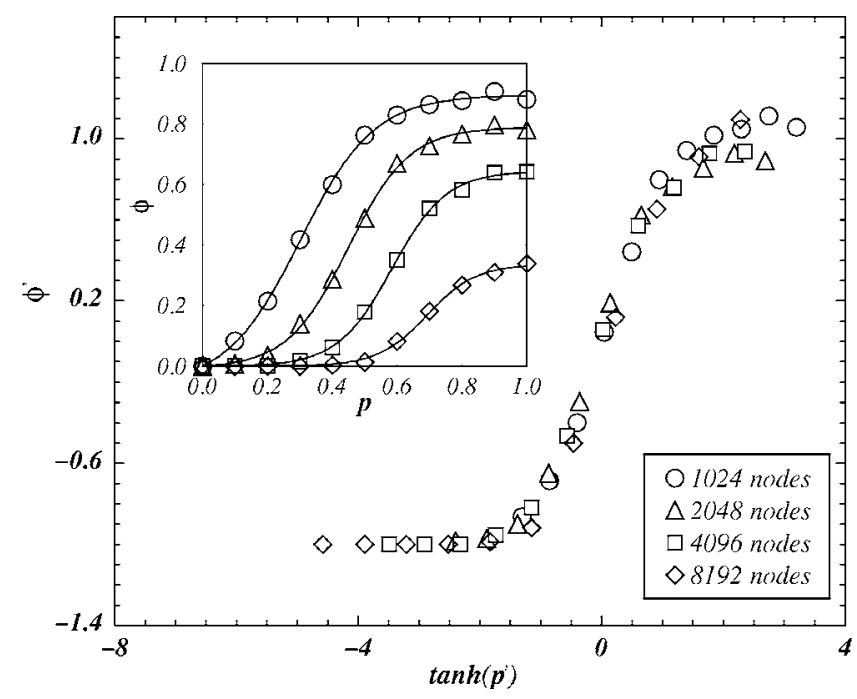

FIG. 3. Renormalized fraction $\phi^{\prime}(N)$ of graphs with periodic time series as a function of the tanh of the renormalized rewiring probability $p^{\prime}(N)$. Inset: Fraction $\phi$ of graphs with periodic time series as a function of the rewiring probability for different network sizes $N$. 


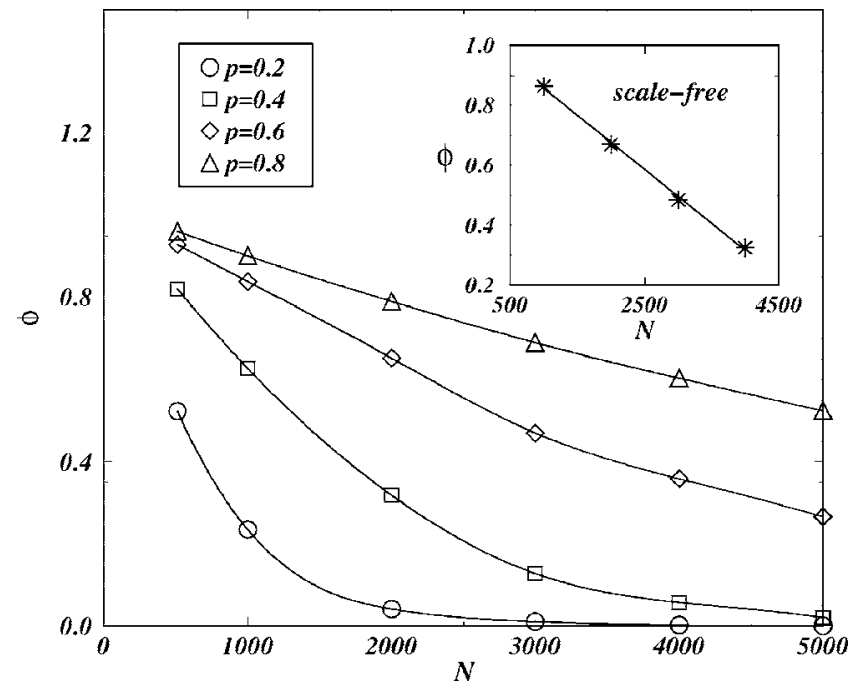

FIG. 4. Fraction $\phi$ of graphs with periodic time series as a function of the size $N$ of the graph for different values of $p$. The inset shows the data for the Barabási-Albert network for $m=3$.

$$
\phi(p, N)=a_{0}\left[\tanh \left(\frac{p}{a_{1}}+a_{2}\right)-\tanh \left(a_{2}\right)\right],
$$

where the parameters $a_{0}, a_{1}$, and $a_{2}$ are obtained through the best nonlinear fit to the data of Eq. (3) for each different value of $N$. In addition, our results suggest that the parameters $a_{i}$ depend only on the system size $N$, since we find their behaviors to be well represented by the relations,

$$
\begin{gathered}
a_{0}(N)=\alpha_{0}+\beta_{0} N, \\
a_{1}(N)=\alpha_{1}+\beta_{1} \ln N, \\
a_{2}(N)=\alpha_{2}+\beta_{2} \ln N,
\end{gathered}
$$

with $\alpha_{0}=0.501, \alpha_{1}=0.476, \alpha_{2}=9.610, \beta_{0}=-4.146 \times 10^{-5}$, $\beta_{1}=-0.036$, and $\beta_{2}=-1.576$. Following this approach, we can rescale the variables $\phi$ and $p$ as $\phi^{\prime}=\phi / a_{0}+\tanh \left(a_{2}\right)$ and $p^{\prime}=p / a_{1}+a_{2}$, respectively, to show that all data can be collapsed on the top of each other as displayed in Fig. 3. From the inset in Fig. 3 we can also see that the degree of periodicity $\phi$ decays with increasing size $N$ of the network for a fixed value of $p>0$.

As can be seen from Fig. 3, there exists a $N_{0} \approx 32000$ defined through $\phi^{\prime}\left(N_{0}\right)=0$ above which the fraction $\phi$ of graphs with periodic signals is zero, $N_{0}$ being the largest network size still showing periods.

In Fig. 4 we show that the degree of periodicity $\phi$ decays with the increase of the network size $N$, and that this effect becomes more pronounced the smaller the value of $p$.

The length of a period is the minimum number of time steps for which the time series is repeated. Figure 5 shows in double-logarithmic scale the average period length as a function of the size $N$ of the network for $N=128,256,512,1024$, and 2048 for a fixed value of $p=0.9$. The average period is defined here as the average over only those configurations that displayed periodic behavior. For each value of $N$, we average over 1000 networks from series that have a total

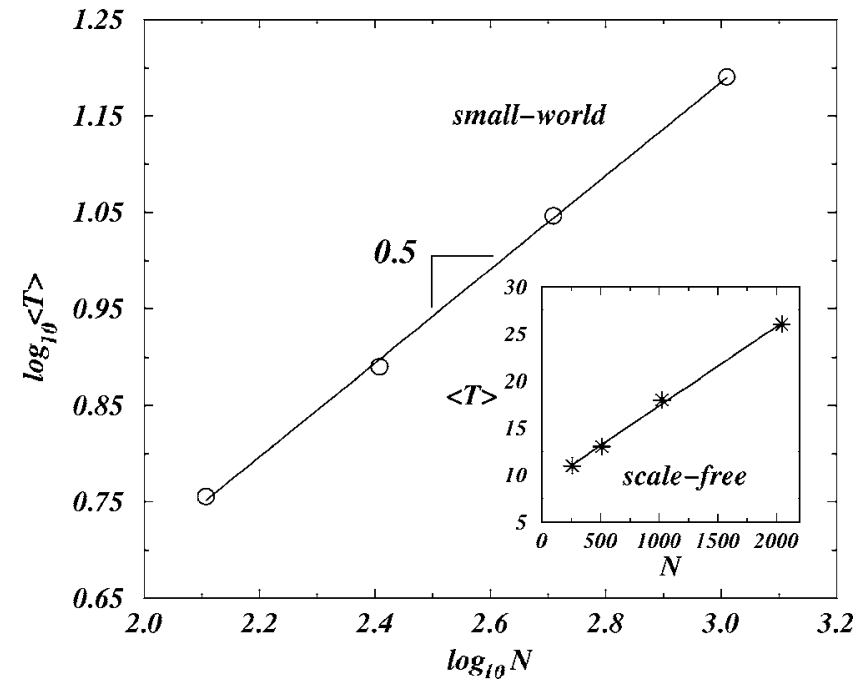

FIG. 5. Double-logarithmic plot of the average period length for the Watts-Strogatz network as function of the size $N$ of the network averaged over 1000 networks for $p=0.9$. The inset shows the data for the Barabási-Albert network for $m=3$.

length of 16384 time steps, the last 1024 of which were analyzed. We see that the average period $\langle T\rangle$ increases with the size like a power law, with an exponent that is approximately equal to $1 / 2$, i.e., $\langle T\rangle \propto \sqrt{N}$.

In the insets of Figs. 4 and 5 we see the corresponding data for a Barabási-Albert network with $m=3$. Here the fraction of graphs having periodic signals decreases with the system size $N$ linearly and at around $N_{0} \approx 6000$ it becomes zero so that for larger sizes no periods can be found. The average period length increases linearly with the size: $\langle T\rangle$ $\propto N$.

In conclusion, we have shown that a simple neural model on Watts-Strogatz and Barabási-Albert networks can generate periodic activity signals, but only if the networks are not too large. These cycles become more frequent if one has more long-range connections, and the length of their period increases like the number of neurons or its square root for scale-free or small-world networks, respectively. Since the brain is huge one would therefore expect the present mechanism to be relevant in only very small fractions of the brain. In particular one can imagine that our discovery is important to explain periodic signals for instance in pacemakers, nervous systems of lower animals, or other very small units of neurons. The similarity of the results for the two types of network also suggests that the dynamic behavior of the system is only weakly dependent on the number of connections and therefore they do not qualitatively affect the conclusions of our paper. Our results have shown that periodic activity may be induced in a network by making it less ordered while extremely ordered structures lead to aperiodic (chaotic) activity, a somewhat surprising and counterintuitive finding.

The brain is one of the big challenges of our century and still full of mysteries and contradictions. From EEG and direct measurements with electrodes we know that cycles of firing do appear. Their role and their origin are still not clear. Of course they are only finite in length and a perfect periodicity has also not yet been confirmed. Our finding suggests at 
least one mechanism that might explain their origin. We see that on small scales the network complexity, be it scale-free or small world, can spontaneously generate periodic signals. We have evidence that real neural networks do have both aspects. In particular, small-world topology has been experimentally evidenced. Small units of neurons could be the nucleus that generates periodic signals using the mechanism found in our work. How they interact with the rest of the brain is not clear. As they stand our results concern small units of neurons and an experimental verification could be imagined with brains of lower animals or with in vitro cultures. One direct application could be the pacemaker of the heart which is indeed a neural net providing a perfectly periodic signal over very long times.

We thank the CNPq, CAPES, FINEP, FUNCAP, and the Max-Planck Foundation for financial support.
[1] D. J. Watts and S. Strogatz, Nature (London) 393, 440 (1998).

[2] D. J. Watts, Small Worlds (Princeton University, Princeton, NJ, 1999).

[3] M. E. J. Newman and D. J. Watts, Phys. Lett. A 263, 341 (1999).

[4] O. Shefi, I. Golding, R. Segev, E. Ben-Jacob, and A. Ayali, Phys. Rev. E 66, 021905 (2002).

[5] J. M. Beggs and D. Plenz, J. Neurosci. 23, 11167 (2003).

[6] S. Maslov, M. Paczuski, and P. Bak, Phys. Rev. Lett. 73, 2162 (1994).

[7] L. F. Lago-Fernandez, R. Huerta, F. Corbacho, and J. A. Sigüenza, Phys. Rev. Lett. 84, 2758 (2000).

[8] L. F. Lago-Fernandez, F. Corbacho, and R. Huerta, Neural Networks 14, 687 (2001)

[9] H. Hong, M. Y. Choi, and B. J. Kim, Phys. Rev. E 65, 026139 (2002).

[10] A. Roxin, H. Riecke, and S. A. Solla, Phys. Rev. Lett. 92,
198101 (2004).

[11] L. de Arcangelis, C. Perrone-Capano, and H. J. Herrmann, Phys. Rev. Lett. 96, 028107 (2006).

[12] L.-A. Barabási and R. Albert, Science 286, 509 (1999).

[13] R. Albert and L.-A. Barabási, Rev. Mod. Phys. 74, 47 (2002).

[14] D. Stauffer, A. Aharony, L. F. Costa, and J. Adler, Eur. Phys. J. B 32, 395 (2003).

[15] G. Grinstein and R. Linsker, Proc. Natl. Acad. Sci. U.S.A. 102, 9948 (2003).

[16] S. Cosenza, P. Crucitti, L. Fortuna, M. Frasca, M. La Rosa, C. Stagni, and L. Usai, Math. Biosci. Eng. 2, 53 (2003).

[17] W. McCulloch and W. Pitts, Bull. Math. Biophys. 5, 115 (1943).

[18] J. E. Moreira, F. W. S. Lima, and J. S. Andrade, Jr., Phys. Rev. E 52, R2129 (1995).

[19] P. Erdös and A. Rényi, Publ. Math. (Debrecen) 6, 290 (1959). 